\title{
MicroRNAs targeting Nicastrin regulate $A \beta$ production and are affected by target site polymorphisms
}

\section{Charlotte Delay ${ }^{1,2,3}$, Véronique Dorval ${ }^{1,2}$, Alice Fok ${ }^{4}$, Benjamin Grenier-Boley ${ }^{3}$, Jean-Charles Lambert ${ }^{3}$, G.-Y. Hsiung ${ }^{4}$ and Sébastien S. Hébert ${ }^{1,2}{ }^{*}$}

'Axe Neurosciences, Centre de Recherche du CHU de Québec, Université Laval, Québec, QC, Canada

2 Département de Psychiatrie et de Neurosciences, Faculté de Médecine, Université Laval, Québec, QC, Canada

${ }^{3}$ Institut Pasteur de Lille, INSERM U744, Université Lille Nord de France, Lille (Nord), France

${ }^{4}$ Division of Neurology, Department of Medicine, University of British Columbia, Vancouver, OC, Canada

\section{Edited by:}

Nicola Maggio, The Chaim Sheba Medical Center, Israel

\section{Reviewed by:}

Gianluca Serafini, Sant'Andrea Hospital, Sapienza University of Rome, Italy

Carlos Bas Orth, University of

Heidelberg, Germany

\section{${ }^{*}$ Correspondence:}

Sébastien S. Hébert, Axe

Neurosciences, Centre de Recherche du CHU de Québec, Université Laval, 2705 boulevard Laurier, P-09800,

Québec, OC G1V 4G2, Canada

e-mail: sebastien.hebert@

neurosciences.ulaval.ca
Despite the growing number of genome-wide association studies, the involvement of polymorphisms in microRNA target sites (polymiRTS) in Alzheimer's disease (AD) remains poorly investigated. Recently, we have shown that AD-associated single-nucleotide polymorphisms (SNPs) present in the $3^{\prime}$ untranslated region ( $3^{\prime}$ UTR) of amyloid precursor protein (APP) could directly affect miRNA function. In theory, loss of microRNA (miRNA) function could lead to risk for AD by increasing APP expression and A $\beta$ peptide production. In this study, we tested the hypothesis that Nicastrin, a $\gamma$-secretase subunit involved in $A \beta$ generation, could be regulated by miRNAs, and consequently affected by $3^{\prime} U T R$ polymorphisms. Bioinformatic analysis identified 22 putative miRNA binding sites located in or near Nicastrin 3'UTR polymorphisms. From these miRNA candidates, six were previously shown to be expressed in human brain. We identified miR-24, miR-186, and miR-455 as regulators of Nicastrin expression, both in vitro and under physiological conditions in human cells, which resulted in altered $A \beta$ secretion. Using luciferase-based assays, we further demonstrated that rs 113810300 and rs 141849450 SNPs affected miRNA-mediated repression of Nicastrin. Notably, rs 141849450 completely abolished the miR-455-mediated repression of Nicastrin. Finally, the rs141849450 variant was identified in 1 out of 511 AD cases but not in 631 controls. These observations set the stage for future studies exploring the role of miRNAs and $3^{\prime} U T R$ polymorphisms in $A D$.

Keywords: Alzheimer's disease, Nicastrin, microRNA, single-nucleotide polymorphism, miR-186, miR-455

\section{INTRODUCTION}

The small, non-coding miRNAs function as important regulators of protein expression (Ambros, 2004). They act by imperfect base-pairing to target messenger RNAs, leading to translational repression, degradation, or both (Pillai, 2005). Important for this action is the seed sequence, located at positions 2-8 within the miRNA sequence. It is now well documented that specific miRNAs are altered in a number of neurodegenerative disorders including Alzheimer's disease (AD; reviewed in Delay et al., 2012). These observations evoke the hypothesis that miRNA dysregulation could directly be implicated in the disease processes, either detrimental or as an attempt of neurons to survive. Moreover, increasing evidence suggests that single-nucleotide polymorphisms (SNP) within miRNA target sites might contribute to disease risk. For instance, the Parkinson's disease-associated rs12720208 SNP is located in the miR-433 target site of Fibroblast growth factor 20, and affects miRNA-mediated repression (Wang et al., 2008). The rs5848 SNP, associated with TDP43-positive frontotemporal dementias, is located in the miR-659 target site of the Progranulin gene, also affecting the miRNA-mediated repression (Rademakers et al., 2008). Previously, we have shown that ADassociated SNPs present in the untranslated region ( $\left.3^{\prime} \mathrm{UTR}\right)$ of APP could directly affect miRNA function (Delay et al., 2011). In theory, loss of miRNA function could contribute significantly to risk for $\mathrm{AD}$ by increasing $\mathrm{APP}$ expression and therefore $\mathrm{A} \beta$ production. $A \beta$ peptides are the major constituents of amyloid (senile) plaques that accumulate in AD brain (Glenner and Wong, 1984).

Another essential player in $A \beta$ production is the $\gamma$-secretase complex, composed of four subunits (i.e., Presenilin, Nicastrin, Aph-1, and Pen2; De Strooper, 2003). Recent studies have documented rare Nicastrin (NCSTN) variants associated with AD (Lupton et al., 2011), whereas studies in mice have shown that increased expression of NCSTN is sufficient to promote AD pathology (Goo etal., 2013). In this study, we evaluated whether miRNAs could modulate NCSTN expression, and whether polymiRTS within the $3^{\prime}$ UTR of the Nicastrin gene could abrogate miRNA function.

\section{MATERIALS AND METHODS PATIENTS}

Cases of AD and controls were participants from two large Canadian cohorts: the Canadian Study of Health and Aging (CSHA) and A Canadian Collaborative Cohort of Cognitive Impairment and Related Dementia (ACCORD). Ethical approval of these studies was obtained from the ethics review board in each of the study centers. Details of each cohort have been published elsewhere 
(Anonymous, 1994; Feldman et al., 2003; Hsiung et al., 2004). The clinical examination was developed in collaboration with the US Consortium to Establish a Registry for Alzheimer's Disease. $\mathrm{AD}$ cases were diagnosed by NINCDS - ADRDA clinical criteria (McKhann et al., 1984), and controls were subjects who remained not cognitively impaired until the end of the study or censorship.

\section{CELL CULTURE}

Human HeLa and HEK293-APPSw cells were cultured in DMEM medium (Invitrogen, Carlsbad, CA, USA) supplemented with $10 \%$ heat-inactivated fetal bovine serum. One day before transfection, HeLa cells were plated at $20 \%$ confluence in six-well or 24-well plates and 250,000 HEK-APPSw cells were plated into sixwell plates. Transfection was performed using Lipofectamine 2000 (Invitrogen, Carlsbad, CA, USA) according to the manufacturer's instructions.

\section{cDNA CONSTRUCTS}

The full-length hNCSTN $3^{\prime}$ UTR was extracted by PCR from SH-SY5Y cells using the following primers: forward: 5'-TTTTTCTAGAGGAGGACCCCAGCTTTTC, reverse 5'-AAAAGGATCCCGTGTGGGATAATCTATTTT, and were cloned using XbaI (New England Biolabs) and BamHI (New England Biolabs) enzymes into the pGL3-promotor vector (Promega, USA). Mutagenesis was performed by TOPgene technologies (Montreal, Quebec, Canada) and validated by sequencing.

\section{LUCIFERASE ASSAY AND PROTEIN ANALYSIS}

HeLa cells were transfected with 25 or $50 \mathrm{nM}$ pre-miRs (Applied Biosystems, USA), $2.5 \mathrm{ng} / \mathrm{cm}^{2}$; pRL control vector, and $50 \mathrm{ng} / \mathrm{cm}^{2}$; pGL3_HSV TK_3'UTR hNCSTN WT or C460T (rs1043329), T623G (rs113810300) or delCA515-516 (rs141849450) mutant variant plasmids. Twenty-four hours post-transfection, cells were lyzed, and luciferase activity was measured according to the manufacturer's instructions (Promega, USA). For western blots, cells were lyzed in RIPA buffer [50 mM Tris $\mathrm{HCl}, 1 \%$ NP40, $0.9 \% \mathrm{NaCl}, 0.25 \%$ Na-deoxycholate, $1 \mathrm{mM}$ EDTA, $1 \times$ proteinase inhibitors (Roche, Basel, Switzerland), $1 \mathrm{mM}$ PMSF, $1 \mathrm{mM}$ $\mathrm{Na}_{3} \mathrm{VO}_{4}$, and $1 \mathrm{mM} \mathrm{NaF}$ ], mixed with LDS sample buffer (Invitrogen, Carlsbad, CA, USA) containing 5\% $\beta$-mercapto-ethanol and boiled at $95^{\circ} \mathrm{C}$ for $8 \mathrm{~min}$. Crude extracts $(10 \mu \mathrm{g})$ were immunoblotted with the Aph-1a [clone B80.2, kind gift from B. De Strooper (Nyabi et al., 2003)], Pen2 (Cell Signaling, clone D2G6), Nicastrin (Cell Signaling, clone D38F9), Presenilin 1

Table 1 | Polymorphisms located in or near miRNA target sites located in the $3^{\prime}$ UTR of $h N C S T N$.

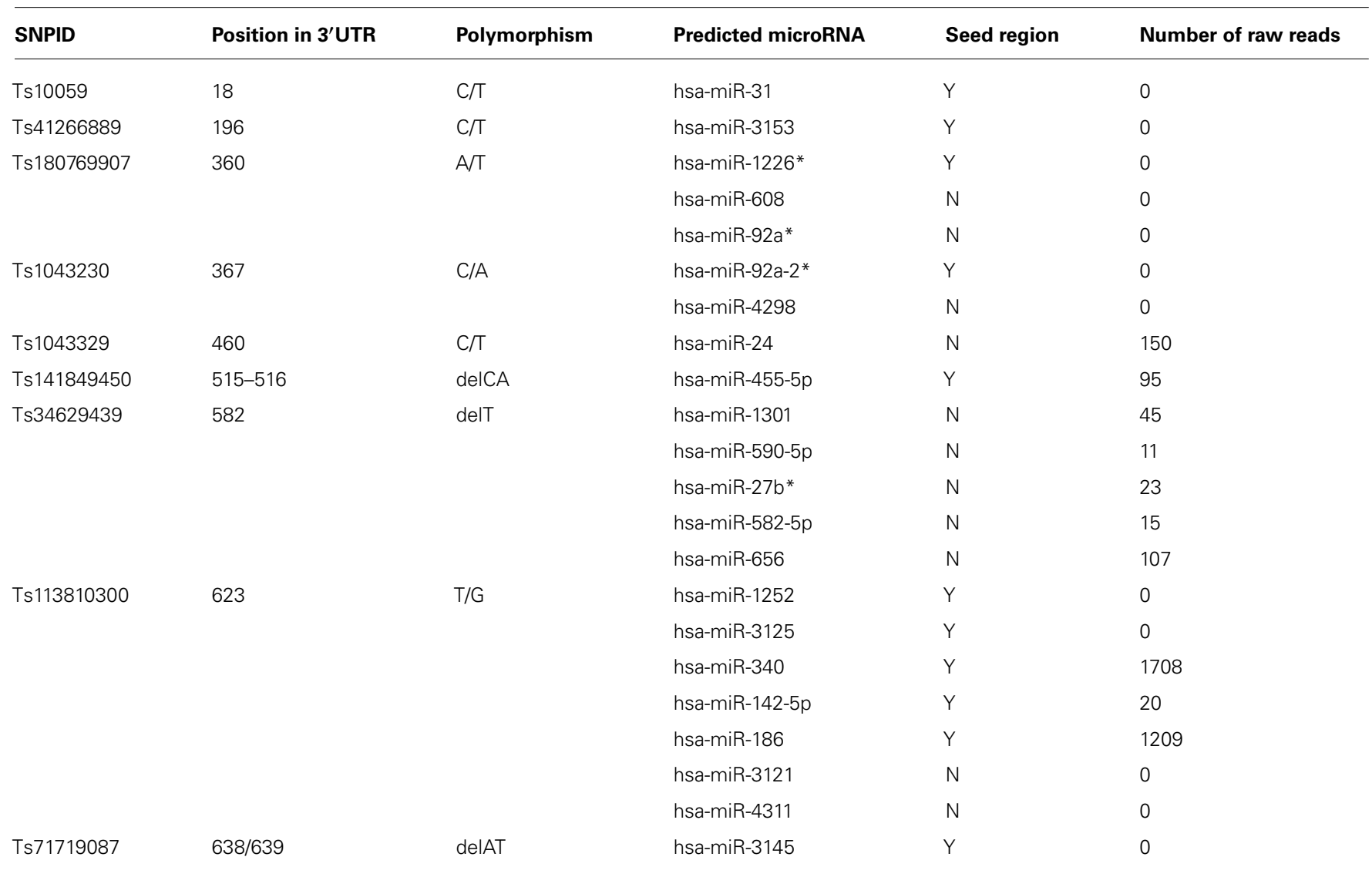

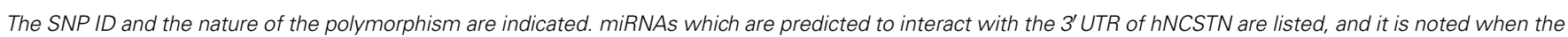

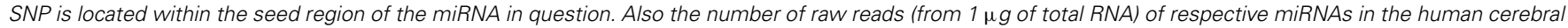
neocortex is noted (see Anonymous, 1994 for details). Y, yes; N, no. 
(Millipore, clone PS1-loop), and Gapdh (Millipore, clone 6c5) antibodies. Membranes were detected using the ECL detection kit (Millipore, Billerica, MA, USA). Quantifications were performed using the Multi Gauge software (FUJIFILM, Minato-ku, Tokyo, Japan).

\section{ELISA}

HEK293-APPSw cells were transfected with $50 \mathrm{nM}$ pre-miRs (Applied Biosystems, USA). Twenty-four hours post-transfection, cell lysates were collected, spun at $1000 \times g$ for $10 \mathrm{~min}$ to remove debris, and supernatants were kept on ice. Human (soluble) $A \beta 1-40$ and $A \beta 1-42$ peptides were measured using $A \beta 40$ and $A \beta 42$ Human ELISA Kits, following the manufacturer's conditions (Life Technologies, Carlsbad, CA, USA).

\section{STATISTICS}

Statistical significance of western blots and luminescence quantifications was determined using one-way ANOVA, two-way ANOVA, or Student's paired $t$-test as indicated in the text. Calculations were made using the GraphPad Prism 5 software.

\section{GENOTYPING}

DNA was extracted from peripheral blood (AutogenFlex STAR, Holliston MA, USA), and SNP genotyping was performed with TaqMan assays in an optimized ABI 7300 (Applied Biosystems). The two SNPs chosen in this study were rs113810300 and rs141849450 in human Nicastrin.

\section{RESULTS}

We first used the National Center for Biotechnology Information (NCBI) database to generate a list of all known SNPs located within the $3^{\prime}$ UTR of human NCSTN (Table 1). In order to identify SNPs that could affect miRNA binding, we used the on-line algorithms TargetScan and microRNA.org (Betel et al., 2008; Garcia et al., 2011). We included both canonical (i.e., perfect seed match) and non-canonical (i.e., containing G:U wobbles or seed mismatches) miRNA binding sites. This analysis resulted
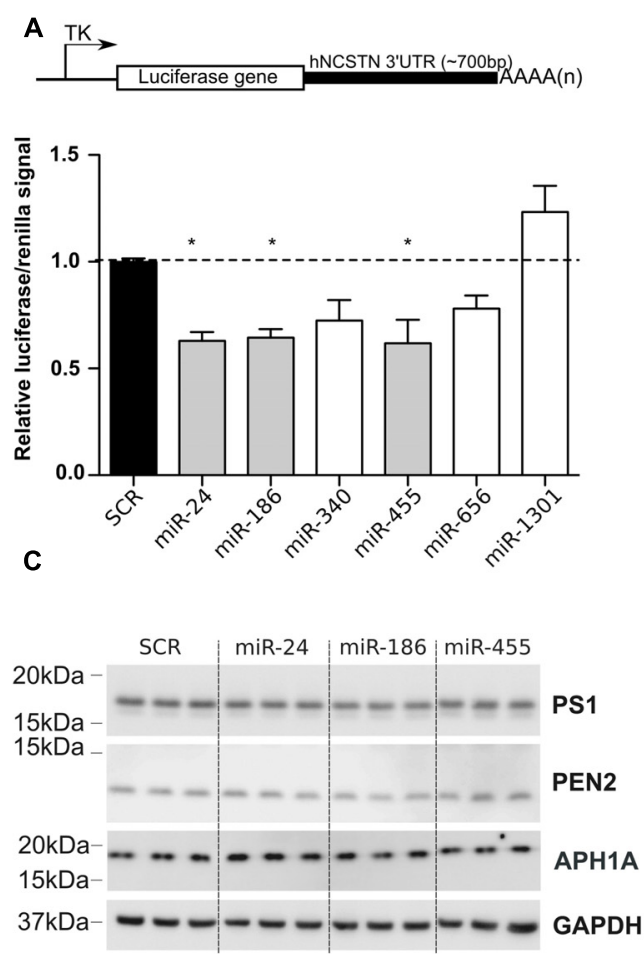

FIGURE 1 | In vitro functional analysis of NCSTN targeting miRNAs. (A) Schematic representation (not to scale) of the luciferase reporter construct used in this study (upper panel). TK; thymidine kinase promoter, AAAA(n); PolyA site, HeLa cells were transfected with $50 \mathrm{nM}$ pre-miRs (as indicated) as well as a reporter construct containing the untranslated region ( $3^{\prime}$ UTR) of hNCSTN. The cells were lyzed $24 \mathrm{~h}$ post-transfection and luciferase signal was measured. Signals were normalization for transfection efficiency and graph represents the luciferase signals compared to the scrambled control (SCR). Statistical significance was assessed by one-way ANOVA with Bonferroni post-hoc test. ${ }^{*} p<0.05$. (B) HeLa cells were transfected with $50 \mathrm{nM}$ pre-miRNAs (as indicated). The cells were lyzed

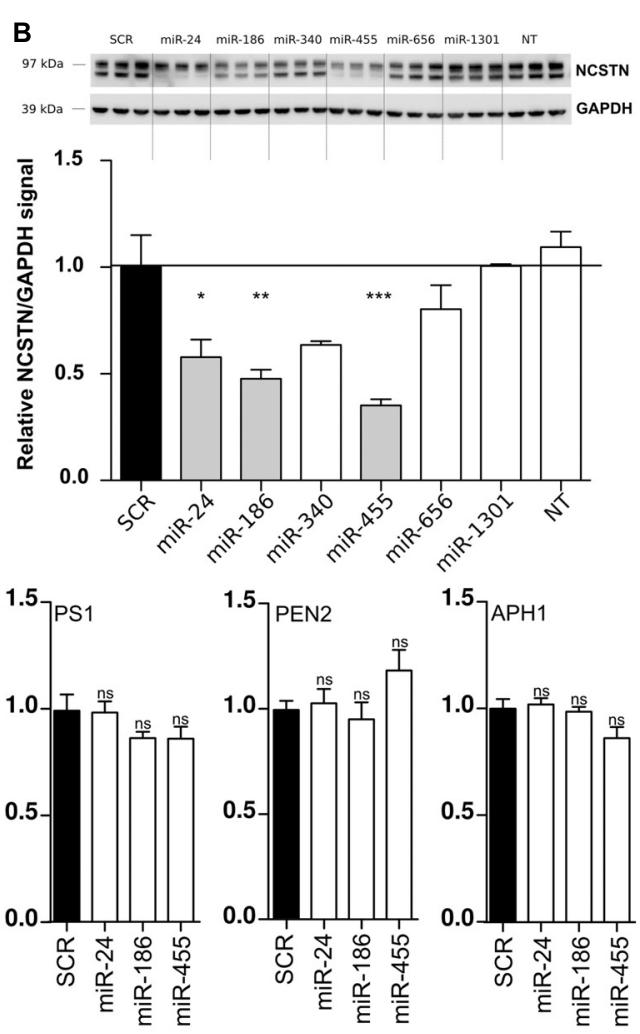

$48 \mathrm{~h}$ post-transfection and western blotting was performed. Representative ( $n=3$, in triplicate) western blots are shown. The ratios of the NCSTN/GAPDH signals are presented (both mature and immature NSCTN). Measurements were normalized to the scrambled control (SCR). Statistical significance was assessed by one-way ANOVA with Bonferroni post-hoc test. ${ }^{*} p<0.05,{ }^{*} p<0.01,{ }^{*}{ }^{*} p<0.001$. (C) HeLa cells were transfected with $50 \mathrm{nM}$ pre-miRNAs (as indicated). The cells were lyzed $48 \mathrm{~h}$ post-transfection and western blotting was performed. Representative ( $n=3$, in triplicate) western blots and quantifications are shown. Statistical significance was assessed by one-way ANOVA with Bonferroni post-hoc test. ns, not significant. 
in a list of 22 miRNAs (Table 1), which we narrowed down to six (i.e., miR-24, miR-186, miR-340, miR-455, miR-656, and miR-1301) based on our previous expression profiling studies in the human cerebral neocortex (45 raw reads cut-off; Hébert et al., 2013). Whether excluded miRNAs are expressed at higher frequency in other brain regions and/or tissues remains to be determined.

To validate our bioinformatic analyses, we cloned the fulllength ( 700 bp) $3^{\prime}$ UTR of human NCSTN into a luciferase reporter vector (Figure 1A), and co-transfected this construct with candidate miRNAs into human HeLa cells. We observed a significant reduction of luciferase expression upon miR-24, miR-186, and miR-455 expression (Figure 1A) compared to a scrambled miRNA (SCR) negative control. We also confirmed the downregulation of endogenous NCSTN after transfection of candidate miRNAs into HeLa cells (Figure 1B). Notably, only miR-186 and miR-455 decreased both the mature and immature forms of NCSTN (Edbauer et al., 2002). While both miR-340 and miR-656 also affected luciferase and endogenous NCSTN expression, this observation did not reach significance. Overexpression of identified NCSTN targeting miRNAs did not significantly affect endogenous Presenilin 1 (PS1), APH1A, or PEN2 expression (Figure 1C). We thus identified miR-24, miR-186, and miR-455 as endogenous regulators of human NCSTN.

In order to determine the functional consequences of miR-24, miR-186, and miR-455 expression on $A \beta$ production, we performed ELISA using HEK293-APPSwe cells. Both miR-186 and miR-455 decreased (soluble) A $\beta 40$ and $A \beta 42$ levels, while miR-24 had a small, nonetheless significant effect on A $\beta 42$ (Figure 2). The observations correlate with our western blot data (Figure 1B), showing that highest NCSTN repression leads to the lowest A $\beta$ secretion.

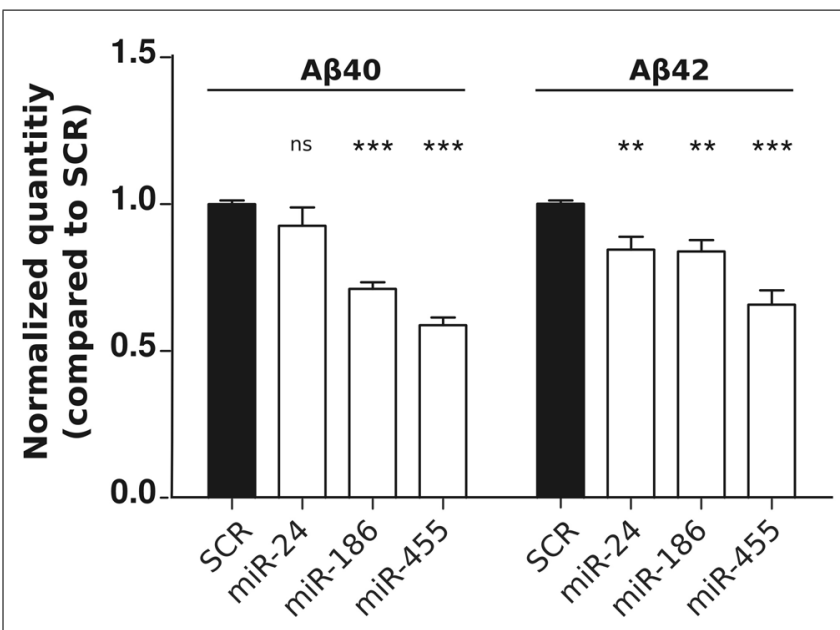

FIGURE 2 | miR-24, miR-186, and miR-455 expression results in decreased $\mathbf{A} \boldsymbol{\beta}$ secretion. Relative quantities of human $A \beta 40$ and $A \beta 42$ peptides measured by ELISA in the culture medium of HEK-APPSwe cells after transfection with $50 \mathrm{nM}$ pre-miRNAs (as indicated). Signals were normalized to the basal A $\beta$ secretion found in cells transfected with SCR miRNAs. Statistical significance was assessed by one-way ANOVA with Bonferroni post-hoc test. ${ }^{*} p<0.05,{ }^{*} p<0.01,{ }^{* *} p<0.001$.
Next, we tested whether these miRNAs were functionally affected by 3'UTR polymorphisms. To address this, we generated human NCSTN 3'UTR luciferase constructs containing the C460T ( $r s 1043329)$, T623G ( $r s 113810300)$, or delCA515-516 ( $r$ 141849450) variants. As before, these mutant vectors were co-transfected with candidate miRNAs and luciferase activity was measured. These screens indicated that the polymorphism C460T, which is located in the $5^{\prime}$ compensatory region of the miRNA binding site, did not significantly affect miR-24 function (Figure 3A). On the other hand, both seed region SNPs T623G and delCA515-516 reduced miR-186 and miR-455-mediated repression, respectively (Figures $3 \mathbf{B}-\mathbf{D}$ ). It is interesting to note that both miR-186 and miR-455 have previously been shown to have an altered expression level in AD cerebrospinal fluid (CSF) samples, which further underscores the possible association between these miRNAs and AD development. Taken together, we identified miR-186 and miR-455, which are functionally affected by polymorphisms.

We finally asked whether identified SNPs could be associated with AD. According to the NCBI dbSNP database (February 2014), the rs113810300 SNP has no reported frequency, while the rs141849450 SNP has a 1/1000 frequency in the European reference panel (1000 Genomes Phase I V3 SHAPEIT2, December 2013). Using imputation from this latter panel, rs141849450 has an estimated frequency of 5.6/1000 in an independent French cohort of more than 2000 individuals (data not shown). To validate and extend these observations, we performed TaqMan SNP genotyping assays in a Canadian patient population of $\mathrm{AD}$ cases and non-demented controls. These screens identified 1 out of $511 \mathrm{AD}$ individuals harboring the rs141849450 deletion mutation. This individual was aged 65 years and was a carrier of the APOE4 (e3/e4) allele. Of notice, the rs141849450 SNP was not present in 631 control. No rs113810300 polymorphism was identified in either groups, as everyone was homozygous T/T. Given the low frequencies of these SNPs in the general population, much larger cohorts are required to determine whether candidate polymorphisms are indeed associated with disease. Nevertheless, we identified at least one SNP co-segregating with an AD patient, which suggests that rare polymiRTS could contribute to risk for disease in a subset of patients. In theory, the rs 141849450 SNP would lead to increased NCSTN expression and A $\beta$ load.

\section{DISCUSSION}

In the current study, we identified a number of brain-expressed miRNAs that could regulate endogenous NCSTN expression and $A \beta$ peptide production. Furthermore, we identified rare $3^{\prime} U T R$ polymorphisms that could affect miRNA repressional activity toward NCSTN, at least in our experimental settings. It has been demonstrated recently that, besides expression variation in miRNAs and genetic variants in miRNA loci, polymiRTS (including both SNPs and small insertions and deletions) are important sources of expression variation of miRNA target genes in humans (Gamazon et al., 2012). Moreover, several SNPs that are in linkage disequilibrium with those that might affect miRNA binding have been identified and are associated with the risk of developing disorders including breast cancer, asthma, and 


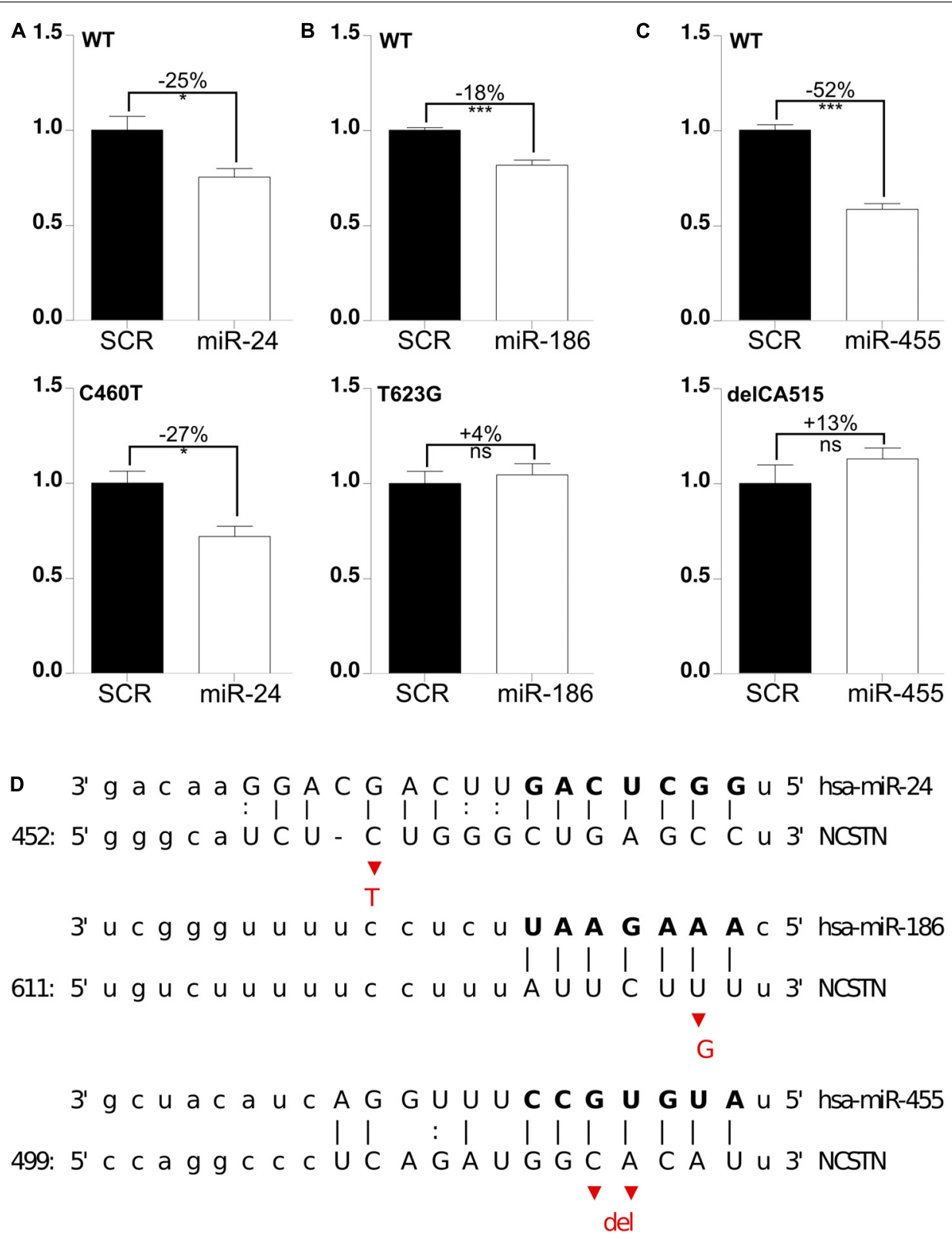

FIGURE 3 | SNP deICA 515-516 and SNP T623G reduce miR-455- and miR-186-mediated NCSTN repression. (A-C) HeLa cells were

transfected with $25 \mathrm{nM}$ pre-miRs (as indicated) as well as a reporter construct containing the WT, delCA 515-516, T623G and C460T mutated $3^{\prime}$ UTR of hNCSTN. The cells were lyzed $24 \mathrm{~h}$ post-transfection and luciferase signal was measured. After normalization for transfection efficiency, the signals were compared to the SCR control. Representative results ( $n=3$, performed in triplicate) are shown. Statistical significance was assessed by Student's paired $t$-test $\left({ }^{*} p<0.05,{ }^{* *} p<0.001\right.$, ns, not significant). SCR; scrambled. (D) Schematic representation of base pair matching between miRNAs and the $3^{\prime}$ UTR of hNCSTN. The seed region of the miRNAs is indicated. The red bases represent the SNPS C460T (upper panel), T623G (middle panel), and delCA 515-516 (lower panel).
Parkinson's disease (Richardson et al., 2011; Thomas et al., 2011). These observations provide significant evidence for the role of genetic-variation-mediated regulation by miRNAs in the etiology of a broad spectrum of human complex phenotypes, including neurodegenerative diseases, metabolic traits, and autoimmune disorders. However, whether the SNPs identified in this study could also confer risk for $\mathrm{AD}$ remains an interesting possibility but will require extensive follow-up studies in larger cohorts of patients. Nevertheless, these observations strengthen the potential role of polymiRTS in neurodegenerative disorders such as AD.

There are some caveats associated with our study. For instance, the luciferase reporter system, although a robust, quantitative method of analysis, has limited clinical relevance. Indeed, the miRNome of immortalized cell lines and a human brain cell are definitely different, making it difficult to account for 
compensatory or cumulative effects by other miRNAs on the same miRNA target site. Secondly, the basal levels of the miRNA of interest as well as the epigenetics of the region at study might be different between brain and cell lines, possibly resulting in different responses. Validation in vivo would be more accurate but would require extensive screening (gene sequencing) studies in patients with $\mathrm{AD}$. Unfortunately, our Canadian patient samples were limited to DNA, so we could not perform in vivo Nicastrin expression studies on mRNA. Another alternative would be to generate multiple knock-in cell lines or animals (e.g., mice) harboring candidate polymorphisms within the endogenous NCSTN $3^{\prime}$ UTR, which extends the current scope of this study. It should be noticed that current mouse models expressing human NCSTN do not contain the (full) $3^{\prime}$ UTR. Moreover, it remains uncertain if the identified miRNAs, although expressed in human brain, are similarly expressed in the mouse brain. Nevertheless, we do provide functional data in human cells which is in line with our main hypothesis that specific miRNAs can regulate $A \beta$ production.

What are the clinical implications of these findings? As mentioned above, this is a notoriously difficult question to answer given the low frequencies of identified SNPs (e.g., 5/1000 for rs141849450) in the general population. These results are somewhat expected and consistent with the notion that functional 3'UTR polymorphisms are uncommon, particularly in the seed region (Chen and Rajewsky, 2006; Saunders et al., 2007; Yu et al., 2007), likely because of evolutionary pressure. They are nevertheless important for human disease, as polymiRTS have been associated with a wide range of diseases, including neurodegenerative disorders such as Parkinson's disease (Wang et al., 2008). Thus, larger cohorts are required to determine whether candidate polymorphisms might be associated with $\mathrm{AD}$. Of notice, we also explored the frequency and possible correlation between $A \beta$ load and the rs141849450 Nicastrin variant in a large French (European) cohort. Unfortunately, we could not conclude anything due to the lack of SNP-bearing subjects (unpublished observations). We do, however, provide experimental validation that the rs141849450 Nicastrin variant exists in at least one AD patient (Canadian cohort). This is important since not based only on prediction algorithms and imputation values. Whether this has clinical relevance is interesting, but it requires extensive follow-up studies in much larger cohorts of patients.

In conclusion, we identified NCSTN-targeting miRNAs (miR24, miR-186, and miR-455) that could decrease $A \beta$ secretion. MiR-186 and miR-455 were previously shown to be altered in $\mathrm{AD}$ CSF samples, and we demonstrated that the miRNAmediated repression of NSCTN mRNA is altered by the presence of SNPs rs113810300 and rs141849450. Our data provide additional proof of principle that polymiRTS could contribute to neurodegenerative disorders, and that miR-186 and miR-455 could be important players in $\mathrm{AD}$ pathology. We also stress the importance of including non-translated regions in genome-wide association studies.

\section{AUTHOR CONTRIBUTIONS}

Charlotte Delay, G.-Y. Hsiung, Sébastien S. Hébert: design of the study. Charlotte Delay, Véronique Dorval, Alice Fok: experiments. Benjamin Grenier-Boley, Jean-Charles Lambert, Charlotte
Delay: statistical analyses. Charlotte Delay, Sébastien S. Hébert: manuscript writing. All authors read and approved the final manuscript.

\section{ACKNOWLEDGMENTS}

The authors thank Claudia Goupil for technical expertise. This work was supported by the CIHR and the Alzheimer Society of Canada, including a fellowship for Charlotte Delay.

\section{REFERENCES}

Ambros, V. (2004). The functions of animal microRNAs. Nature 431, 350-355. doi: 10.1038 /nature 02871

Anonymous. (1994). Canadian study of health and aging: study methods and prevalence of dementia. CMAJ 150, 899-913.

Betel, D., Wilson, M., Gabow, A., Marks, D. S., and Sander, C. (2008). The microRNA.org resource: targets and expression. Nucleic Acids Res. 36, D149-D153. doi: 10.1093/nar/gkm995

Chen, K., and Rajewsky, N. (2006). Natural selection on human microRNA binding sites inferred from SNP data. Nat. Genet. 38, 1452-1456. doi: 10.1038/ng1910

Delay, C., Calon, F., Mathews, P., and Hébert, S. S. (2011). Alzheimerspecific variants in the $3^{\prime} \mathrm{UTR}$ of Amyloid precursor protein affect microRNA function. Mol. Neurodegener. 6, 70. doi: 10.1186/17501326-6-70

Delay, C., Mandemakers, W., and Hébert, S. S. (2012). MicroRNAs in Alzheimer's disease. Neurobiol. Dis. 46, 285-290. doi: 10.1016/j.nbd.2012.01.003

De Strooper, B. (2003). Aph-1, Pen-2, and Nicastrin with Presenilin generate an active gamma-Secretase complex. Neuron 38, 9-12. doi: 10.1016/S08966273(03)00205-8

Edbauer, D., Winkler, E., Haass, C., and Steiner, H. (2002). Presenilin and nicastrin regulate each other and determine amyloid beta-peptide production via complex formation. Proc. Natl. Acad. Sci. U.S.A. 99, 8666-8671. doi: 10.1073/pnas.132277899

Feldman, H., Levy, A. R., Hsiung, G.-Y., Peters, K. R., Donald, A., Black, S. E., et al. (2003). A Canadian cohort study of cognitive impairment and related dementias (ACCORD): study methods and baseline results. Neuroepidemiology 22, 265-274. doi: 10.1159/000071189

Gamazon, E. R., Ziliak, D., Im, H. K., LaCroix, B., Park, D. S., Cox, N. J., et al. (2012). Genetic architecture of microRNA expression: implications for the transcriptome and complex traits. Am. J. Hum. Genet. 90, 1046-1063. doi: 10.1016/j.ajhg.2012.04.023

Garcia, D. M., Baek, D., Shin, C., Bell, G. W., Grimson, A., and Bartel, D. P. (2011). Weak seed-pairing stability and high target-site abundance decrease the proficiency of lsy-6 and other microRNAs. Nat. Struct. Mol. Biol. 18, 1139-1146. doi: $10.1038 / \mathrm{nsmb} .2115$

Glenner, G. G., and Wong, C. W. (1984). Alzheimer's disease: initial report of the purification and characterization of a novel cerebrovascular amyloid protein. Biochem. Biophys. Res. Commun. 120, 885-890. doi: 10.1016/S0006291X(84)80190-4

Goo, J. S., Kim, Y. B., Shim, S. B., Jee, S. W., Lee, S. H., Kim, J. E., et al. (2013). Nicastrin overexpression in transgenic mice lnduces aberrant behavior and APP processing. Mol. Neurobiol. 48, 232-243. doi: 10.1007/s12035-013-8453-3

Hébert, S. S., Wang, W.-X., Zhu, Q., and Nelson, P. T. (2013). A study of small RNAs from cerebral neocortex of pathology-verified Alzheimer's disease, dementia with lewy bodies, hippocampal sclerosis, frontotemporal lobar dementia, and nondemented human controls. J. Alzheimers Dis. 35, 335-348. doi: 10.3233/JAD122350

Hsiung, G.-Y., Sadovnick, A. D., and Feldman, H. (2004). Apolipoprotein E epsilon4 genotype as a risk factor for cognitive decline and dementia: data from the Canadian Study of Health and Aging. CMAJ 171, 863-867. doi: 10.1503/cmaj.1031789

Lupton, M. K., Proitsi, P., Danillidou, M., Tsolaki, M., Hamilton, G., Wroe, R., et al. (2011). Deep sequencing of the Nicastrin gene in pooled DNA, the identification of genetic variants that affect risk of Alzheimer's disease. PLOS ONE 6:e17298. doi: 10.1371/journal.pone.0017298

McKhann, G., Drachman, D., Folstein, M., Katzman, R., Price, D., and Stadlan, E. M. (1984). Clinical diagnosis of Alzheimer's disease: report of the NINCDS-ADRDA Work Group under the auspices of Department of Health 
and Human Services Task Force on Alzheimer's Disease. Neurology 34, 939-944. doi: 10.1212/WNL.34.7.939

Nyabi, O., Bentahir, M., Horré, K., Herreman, A., Gottardi-Littell, N., Van Broeckhoven, C., et al. (2003). Presenilins mutated at Asp-257 or Asp-385 restore Pen-2 expression and Nicastrin glycosylation but remain catalytically inactive in the absence of wild type Presenilin. J. Biol. Chem. 278, 43430-43436. doi: 10.1074/jbc.M306957200

Pillai, R. S. (2005). MicroRNA function: multiple mechanisms for a tiny RNA? RNA 11, 1753-1761. doi: 10.1261/rna.2248605

Rademakers, R., Eriksen, J. L., Baker, M., Robinson, T., Ahmed, Z., Lincoln, S. J., et al. (2008). Common variation in the miR-659 binding-site of GRN is a major risk factor for TDP43-positive frontotemporal dementia. Hum. Mol. Genet. 17, 3631-3642. doi: 10.1093/hmg/ddn257

Richardson, K., Lai, C.-Q., Parnell, L. D., Lee, Y.-C., and Ordovas, J. M. (2011). A genome-wide survey for SNPs altering microRNA seed sites identifies functional candidates in GWAS. BMC Genomics 12:504. doi: 10.1186/1471-2164-12-504

Saunders, M. A., Liang, H., and Li, W.-H. (2007). Human polymorphism at microRNAs and microRNA target sites. Proc. Natl. Acad. Sci. U.S.A. 104, 3300-3305. doi: 10.1073/pnas.0611347104

Thomas, L. F., Saito, T., and Sætrom, P. (2011). Inferring causative variants in microRNA target sites. Nucleic Acids Res. 39:e109. doi: 10.1093/nar/gkr414

Wang, G., van Der Walt, J. M., Mayhew, G., Li, Y.-J., Züchner, S., Scott, W. K., et al. (2008). Variation in the miRNA-433 binding site of FGF20 confers risk for
Parkinson disease by overexpression of alpha-synuclein. Am. J. Hum. Genet. 82, 283-289. doi: 10.1016/j.ajhg.2007.09.021

Yu, Z., Li, Z., Jolicoeur, N., Zhang, L., Fortin, Y., Wang, E., et al. (2007). Aberrant allele frequencies of the SNPs located in microRNA target sites are potentially associated with human cancers. Nucleic Acids Res. 35, 4535-4541. doi: 10.1093/nar/gkm480

Conflict of Interest Statement: The authors declare that the research was conducted in the absence of any commercial or financial relationships that could be construed as a potential conflict of interest.

Received: 07 May 2014; accepted: 02 July 2014; published online: 18 July 2014.

Citation: Delay C, Dorval V, Fok A, Grenier-Boley B, Lambert J-C, Hsiung G-Y and Hébert SS (2014) MicroRNAs targeting Nicastrin regulate A $\beta$ production and are affected by target site polymorphisms. Front. Mol. Neurosci. 7:67. doi: 10.3389/fnmol.2014.00067

This article was submitted to the journal Frontiers in Molecular Neuroscience. Copyright (C) 2014 Delay, Dorval, Fok, Grenier-Boley, Lambert, Hsiung and Hébert. This is an open-access article distributed under the terms of the Creative Commons Attribution License (CC BY). The use, distribution or reproduction in other forums is permitted, provided the original author(s) or licensor are credited and that the original publication in this journal is cited, in accordance with accepted academic practice. No use, distribution or reproduction is permitted which does not comply with these terms. 